ene

\title{
ESTILOS DE VIDA RELACIONADOS CON FACTORES DE RIESGO CARDIOVASCULAR EN ESTUDIANTES CiEnCIAS DE LA SALUD
}

\section{LIFESTYLES RELATED TO CARDIOVASCULAR RISK FACTORS IN STUDENTS HEALTH SCIENCES}

Mariela Elisa Suarez Villa, Maloris De Jesús Navarro Agamez, Daniela Rocio Caraballo Robles, Laura Vanessa López Mozo, Andrea Carolina Recalde Baena

Universidad Simón Bolivar

Colombia 


\section{Resumen}

Los estilos de vida poco saludables en estudiantes universitarios son comportamientos de riesgo para la salud. El objetivo fue determinar los estilos de vida relacionados con factores de riesgo cardiovascular en Estudiantes de Ciencias de Salud. Estudio de tipo descriptivo transversal, población de 3070 estudiantes, el muestreo probabilístico estratificado por afijación proporcional con 294 estudiantes, se recolecto la información por medio de encuesta estructurada, se tomaron medidas antropométricas como talla, peso y circunferencia abdominal. En conclusión, la mayoría de estudiantes de las ciencias de la salud de la universidad de Barranquilla presentan estilos de vida que son factores de riesgo enfermedades cardiovasculares

Palabras claves: Estilo de vida; Adolescente; Factores de riesgo; Enfermedades cardiovasculares; Estudiantes; Ciencias de la Salud.

\section{Abstract}

Unhealthy lifestyles in college students are risky health behaviors. The objective was to determine the lifestyles related to cardiovascular risk factors in Health Science Students. Cross-sectional descriptive study, population of $\mathbf{3 0 7 0}$ students, probabilistic sampling stratified by proportional affixation with 294 students, information was collected through a structured survey, anthropometric measures were taken such as height, weight and abdominal circumference. In conclusion, most students of the health sciences at the Simón Bolívar University have lifestyles that are risk factors for cardiovascular diseases.

Keywords: Lifestyle; Adolescent; Risk factor's; cardiovascular diseases; Students; Health Sciences. 


\section{INTRODUCCIÓN}

Los hábitos saludables se refieren al modo de vida de una persona o grupo de individuos en el que intervienen particularidades individuales, patrones de conductas personales que incluye el estado de vida o de salud de manera general y los aspectos socioculturales (1). El predominio de estilos de vida poco saludable en estudiantes universitarios se presenta debido a que se encuentran en una etapa en la que se abandonan ciertos hábitos sanos; está ampliamente demostrado que determinadas conductas de riesgo como el tabaquismo, dieta poco saludable, conducta sedentaria o excesivo consumo de alcohol, son factores de riesgo que pueden contribuir de forma independiente a incrementar la morbimortalidad (2).

En la adolescencia se aprenden conductas que tienen importantes repercusiones sobre la salud; las cuales se fortalecen en la juventud y son difíciles de modificar en la edad adulta. Por ello la enseñanza de hábitos de vida saludables ocupa un papel muy importante, debido a que es en esta etapa donde se deben adquirirse conocimientos, habilidades y actitudes, que permitan el desarrollo de comportamientos sanos. Está plenamente comprobado que los principales problemas de salud en los jóvenes están relacionados con sus estilos de vida (3).

Las principales causas de muerte provienen de estilos de vida y conductas poco saludables que conllevan a presentar enfermedades crónicas no transmisibles (ECNT). Los factores de riesgo relacionados con el comportamiento, aumentan la prevalencia de estas enfermedades; en consecuencia las enfermedades cardiovasculares son el resultado de es$^{-}$ tilos de vida poco saludable; cada vez se hacen más frecuentes a temprana edad y se consideran un problema de salud pública $(4,5)$.

Los factores de riesgo asociados a estilos de vida poco saludables, traen como consecuencia la obesidad, la hipertensión arterial, las dislipidemias y la hiperglucemia (6). Las enfermedades cardiovasculares causan aproximadamente dos terceras partes de las defunciones que ocurren en todo el mundo, el $80 \%$ de las cuales se registran en los países de ingresos bajos y medianos (7).

En Cuba se evidencia que los estudiantes universitarios consumen alcohol en un $79 \%$ siendo las mujeres quienes consumen más que los hombres; con respecto al tabaco el $27 \%$ de los estudiantes lo consume sin diferencia de género. En ambos sexos el consumo de drogas ilegales fue de $9 \%$, el $47 \%$ del total de estudiantes realizaba activi- 
dad física y el $37 \%$ consumía una dieta adecuada (8).

En otro estudio realizado en universitarios chilenos, se evidencia que solo un $7 \%$ consumen frutas y $27 \%$ verduras; además presentaron un elevado consumo de alcohol, en cuanto al tabaco el $33,5 \%$ de los hombres consume lo consume y las mujeres en un $28,3 \%$; con referente a la actividad física el 17,8 $\%$ de los hombres la realizan con más frecuencia (9).

Estudio realizado en Universidad Nacional de Colombia evidenció que el $29 \%$ de los estudiantes diariamente consumen productos de paquete y el $26 \%$ ingieren gaseosa, destacándose que el $40 \%$ de los hombres ingieren gaseosas con una frecuencia de 2 a 3 veces por semana; en cuanto al consumo de comidas rápidas, el $11 \%$ lo hacía 2 a 3 veces por semana. Además, el $21 \%$ de los estudiantes consumía alimentos fritos a diario y el $40 \%$ lo hacía 2 a 3 veces por semana, siendo los hombres quienes más consumían este tipo de alimentos (7). Todos estos datos corroboran, que los estilo de vida saludable están relacionado con aspectos como, una dieta equilibrada, el mantenimiento del peso corporal, la práctica habitual de actividad física, y la renuncia del consumo de alcohol y tabaco entre otros; cuando los comportamientos no saludables no se corrigen a tiempo traerán complicaciones que conllevan a padecer enfermedades cardiovasculares (10).

Los estudiantes universitarios están en una fase donde es primordial poner en práctica hábitos saludables en todos los ambientes donde se desenvuelvan, como son el social, familiar y laboral, para contribuir de esta manera a disminuir los factores riesgos cardiovasculares que se puedan presentar; todo ello enfocado en la prevención, para la minimización de enfermedades crónicas no transmisibles (11). Además; cabe resaltar la importancia que juega la enfermería, puesto que son las enfermeras el profesional de la salud encargado de planear, dirigir y aplicar los programas de promoción de la salud, de manera específica el programa de riesgo cardiovascular para la adquisición de hábitos saludables que redunden en el mantenimiento de la salud (10). Por ello es importante concientizar a los estudiantes universitarios del riesgo que generan los inadecuados hábitos de estilo de vida sobre la salud. El objetivo de la investigación es determinar los estilos de vida relacionados con factores de riesgo cardiovascular en estudiantes de la Ciencia Salud. 


\section{MÉTODO}

Estudio cuantitativo, de tipo descriptivo transversal se realizó muestreo probabilístico estratificado por afijación proporcional. La población estuvo conformada por 3070 estudiantes de Ciencias de la Salud de una universidad de Barranquilla en 2018 , se dividió la población en cuatro estratos que correspondieron a los programas de pregrado de las Ciencias de la Salud; con la fórmula determinación de la muestra, se aplicó a cada estrato teniendo en cuenta un nivel de confianza de $95 \%$ y un margen de error del $5 \%$, por último se realizó en cada estrato la afijación proporcional al $30 \%$ dando como resultado una muestra por programa (Medicina 87, Enfermería 80, Fisioterapia 74 e Instrumentación quirúrgica 53); para un total de 294 estudiantes universitarios de las Ciencias de la Salud.

Los criterios de inclusión fueron; solo los estudiantes de la universidad de Barranquilla matriculados en los programas de la Facultad de Ciencias de la salud (Medicina, Enfermería, Fisioterapia e Instrumentación Quirúrgica). Se excluyeron los estudiantes de los programas de post grados y estudiantes de las ciencias de la salud de otras universidades.

Para la recolección de la información, se realizó una encuesta estructurada con el instrumento de la OMS para la vigilancia de los factores de riesgo de las enfermedades crónicas" (STEPS) (12). La cual contenía 58 preguntas abierta, cerradas y de escala de Likert, en el cual se evaluaron las variables sociodemográficas, la alimentación, el nivel de acti vidad física y el consumo de tabaco y alcohol, el instrumento fue validado por expertos y además, se realizó una prueba piloto. Teniendo en cuenta los aspectos éticos esta investigación es clasificada sin riesgo según la resolución 8430 de 1993 (13), también se tuvo en cuenta los principios éticos para las investigaciones médicas en seres humano postuladas en la declaración de Helsinki (14). Para efectuar la presentación y análisis de la información se utilizó la base de datos mecánica IBM SPSS STATISTICS 25.

\section{REsultados}

En cuanto a las características sociodemográficas predominó el sexo femenino con un porcentaje del $68 \%$, prevaleciendo las edades entre 20 a 24 años representada en un $52 \%$, la procedencia fue la urbana con el $75 \%$, y el $84 \%$ de los estudiantes son solteros ver Tabla 1.

En cuanto a los factores de riesgo relacionados con los estilos de vida, se evidencia el consumo de tabaco en los programas de Fisioterapia e Instrumen- 
tación Quirúrgica en un $8,1 \%$ y $7,5 \%$ respectivamente. Con respecto al alcohol los programas que lo consumen son: Fisioterapia en un $35,1 \%$ y Medicina en un $23,3 \%$. Los programas que no realizan ningún tipo de actividad física fueron: Enfermería en un 56,3\%, Fisioterapia en un $54,1 \%$ y Medicina con un $47,1 \%$. Ver tabla 2.

Además se evidencia que los programas que consumen comidas ricas en grasas son: Instrumentación Quirúrgica y Enfermería con porcentajes de $39,6 \%$ y $35 \%$ respectivamente, con respecto al consumo de gaseosa, los programas que la ingieren son Instrumentación Quirúrgica en un 30\% y Medicina en un $21,6 \%$; en cuanto a las bebidas energizantes los programas que la consumen son Enfermería e Instrumentación Quirúrgica con porcentajes de $30 \%$ y $28,3 \%$ respectivamente, y los programas que consumen comidas rápidas son Instrumentación Quirúrgica en un $32,1 \%$ y Fisioterapia con el $18,9 \%$. Ver tabla 3.

\section{Discusión}

Los estilos de vida saludable relacionados con los factores de riesgo cardiovascular en jóvenes universitarios tanto en de las ciencias de la salud como en otras ciencias se han convertido en una problemática de salud a nivel mun- dial; según la Organización Mundial de la Salud (OMS), las enfermedades cardiovasculares (ECV) son la principal causa de muerte a nivel mundial. En el 2012 hubo 38 millones de vidas perdidas por $E C V$, el $42 \%$ fueron defunciones prematuras. Se calcula que para el año 2030, morirán cerca de 23,6 millones de personas por ECV (15).

En cuanto a las características sociodemográficas predominó el sexo femenino con un porcentaje del $68 \%$, el rango de edad más frecuente se encontró entre los 20 y 24 años con un 52\%, y el $84 \%$ de los estudiantes son solteros; datos que coinciden con el estudio de Mollinedo, Trejo at al; (16), en el cual la mayoría de los participantes fueron del sexo femenino con una edad promedio de 20 años $(D E=2,2)$, en su mayoría solteros.

Con respecto a los factores de riesgo relacionados con el tabaquismo los estudiantes de ciencias de la salud fuman, el $8,1 \%$ de fisioterapia el 7,5\% los de Instrumentación Quirúrgica, datos similares a los encontrados por Salazar et al (17). Además, se evidencia que los estudiantes de Fisioterapia y Medicina consumen algunas veces alcohol en un $35,1 \%$ y $23,3 \%$, mostrando similitud con el estudio realizado por Salazar et al, donde el consumo de alcohol fue de $26 \%$ (17). 
En cuanto a la actividad física los programas de Enfermería, Fisioterapia y Medicina no realizan actividad física con un porcentaje de $56,3 \%, 54,1 \%$ y $47,1 \%$ respectivamente; datos similares al estudio de Acuña, donde los estudiantes presentaron una prevalencia de sedentarismo de $42,77 \%$, mostrando que los estudiantes de enfermería son los más sedentarios (18). Otro estudio realizado en una universidad al norte de la ciudad de Lima-Perú a la evaluación del nivel de actividad física, arrojo que el $25,9 \%$ resultaron con actividad física baja (19).

Referente a los hábitos alimentarios, los estudiantes consumen comidas ricas en grasas principalmente son los programas de Instrumentación Quirúrgica y Enfermería en un promedio de 37\%; además siempre consumen gaseosa los programas de Instrumentación Quirúrgica y Medicina en promedio el $25.8 \%$, el programa que consume con frecuencia comidas rápidas es Instrumentación Quirúrgica en un $32,1 \%$, datos que presentan similitud con el estudio de Fajardo et al (20), donde se evidencio en estudiantes universitarios el consumo de embutidos y comidas ricas en grasa fue en un $48,6 \%$, haciendo énfasis en que los estudiantes consumen frecuentemente comidas rápidas en un $37.5 \%$ del total de la población; en cuanto a las bebidas energizantes frecuentemente lo consu- men los programas de Enfermería e Instrumentación Quirúrgica con porcentajes de $30 \%$ y $28,3 \%$, datos contrarios a los resultados del mismo estudio donde solo el $17,6 \%$ consume bebidas energéticas una vez a la semana (20).

La OMS ratificó para la prevención y control de enfermedades no transmisibles el plan de acción 2013-2020 que busca reducir el número de defunciones prematuras por ECNT en un $25 \%$ para el 2025 , por lo que incentiva el abordar los factores de riesgo para estas enfermedades, particularmente en el consumo de tabaco y alcohol, la inactividad física, la ingesta de sal, la hipertensión; todo ello mediante el fomento de prácticas saludables como el consumo de frutas y verduras al menos 5 raciones al día, realizar actividad física al menos 30 minutos todos los días, campañas que ayuden a reducir el consumo de tabaco, alentando a proteger a los no fumadores y persuadiendo a los jóvenes para que dejen de fumar; todo ello para la prevención de los infartos de miocardio y accidentes cerebrovasculares ${ }^{(21)}$

\section{CONCLUSIÓN}

Se concluye que gran parte de los estudiantes de las Ciencias de la Salud de la universidad Simón Bolívar a pesar de ser una población adulta joven, 
presentan estilos de vida que son factores de riesgo que con llevan a enfermedades cardiovasculares, principalmente la inactividad física y la inadecuada alimentación en los programas de Enfermería y Fisioterapia, de igual manera se suma en ellos el consumo de alimentos ricos en grasa.

Por los resultados obtenidos en el presente estudio es importante plantear estrategias de educación en salud que promuevan estilos de vida saludable en la vida cotidiana de los estudiantes las cuales pueden ser abordadas por el servicio de bienestar universitario para minimizar los riesgos modificables y así contribuir a la prevención y control de las enfermedades cardiovasculares. De igual forma es importante la identificación temprana de alteraciones que permitan realizar intervenciones específicas para mejorar el estilo de vida y contribuir con el bienestar de esta población a lo largo del tiempo. Por tanto, también es trascendental sensibilizar sobre la mejora de estilos de vida saludable en población universitaria, haciendo énfasis en actitudes y comportamientos que promuevan estilos de vida que favorezcan la salud cardiovascular puesto que como futuros profesionales de la salud están llamados a ser verdadero ejemplo de motivación, dignos de imitar.

\section{AGRADECIMIENTO}

Se le agradece a la universidad Simón Bolívar de Barranquilla por brindarnos el espacio para la realización de esta investigación con los estudiantes participantes de las Ciencias de la Salud. 


\section{BiBLIOGRAFÍA}

1. Herazo ,Y., Campo, L., García, F., Suarez, M., Méndez, O., y Vásquez, F. (2017) Estilos de vida saludables de niños, niñas y adolescentes de Barranquilla. Salud Uninorte, 33, 419-428.

2. Morales I, Gladys, del Valle R, Carlos, Soto V, Álvaro, y Ivanovic M, Daniza. (2013). Factores de riesgo cardiovascular en estudiantes universitarios. Revista chilena de nutrición, 40(4), 391-396. Recuperado en: https://dx.doi.org/ 10.4067/S0717-75182013000400010

3. Bennásar, M. (2014). Estilos de vida y salud en estudiantes universitarios: la universidad como entorno promotor de la salud. Tesis doctoral no publicada . Institut Universitaria Investigación en Ciències de la Salut, España.

4. World Health Organization. (2013). Información general sobre la hipertensión en el mundo. Una enfermedad que mata en silencio, una crisis de salud pública mundial. Día Mundial de la Salud. De la wold wide web: https://apps.who.int/iris/bitstream/handle/10665/87679/WHO_DCO_WHD_2013.2_spa.pdf;jsessionid=4FD3C74E3EAD5C883DA6A36370E0E184? sequence=1.

5. Padilla-García, C. I., Jaimes-Valencia, M. L., Fajardo-Nates, S., \& Ramos-Franco, A. M. (2014). Factores de riesgo cardiovascular y estilos de vida de estudiantes universitarios. MedUNAB, 17(2), 81-90. Recuperado en: https://revistas.unab.edu.co/index.php/medunab/article/view/2171/1976

6. Cruz-Sánchez, E., Orosio-Méndez, M., CruzRamírez, T., Bernardino-García, A., Vásquez-Domínguez, L. Galindo-Palma, N., \& Grajales-Alonso, I. (2018). Factores de riesgo cardiovascular en estudiantes de enfermería de una universidad pública. Enfermería Universitaria, 13(4). Recuperado en: http://dx.doi.org/10.1016/j.reu. 2016.09.004

7. Patel, P., Ordunez, P., DiPette, D., Escobar, M. C., Hassell, T., Wyss, F., y Angell, S. (2017). Mejor control de la presión arterial para reducir la morbilidad y mortalidad por enfermedades cardiovasculares: Proyecto de Prevención y Tratamiento Estandarizado de la Hipertensión Arterial. Revista Panamericana de Salud Pública, 41, 1. Recuperado en: https://www.scielosp.org/pdf/rpsp/ 2017.v41/1/es

8. Diéguez Martínez, M., Miguel Soca, P. E., Rodríguez Hernández, R., López Báster, J., y Ponce de León, D. (2017). Prevalencia de obesidad abdominal y factores de riesgo cardiovascular asociados en adultos jóvenes. Revista Cubana de Salud Pública, 43, 396-411. Recuperado en: https://www.scielosp.org/article/rcsp/ 2017.v43n3/396-411/pt/\#

9.Durán, S., Crovetto, M., Espinoza, V., Mena, F., Oñate, G., Fernández, M., y Valladares, M. (2017).
Caracterización del estado nutricional, hábitos alimentarios y estilos de vida de estudiantes universitarios chilenos: estudio multicéntrico. Revista médica de Chile, 145(11), 1403-1411. Recuperado en: https://scielo.conicyt.cl/scielo.php? script=sci_arttext\&pid=S0034-9887201700110140 $3 \& \operatorname{lng}=\mathrm{es}$.

10. Gómez Cruz, Z., Landeros Ramírez, P., Romero Velarde, E., \& Troyo Sanromán, R. (2016). Estilos de vida y riesgos para la salud en una población universitaria. Revista Salud Pública y Nutrición, 15(2), 15-21. Recuperado en: http://www.medigraphic.com/pdfs/revsalpubnut/spn-2016/ spn162c.pdf

11. Alarcón, M., Delgado, P., Caamaño, F., Osorio, A., Rosas, M., \& Cea, F. (2015). Estado nutricional, niveles de actividad física y factores de riesgo cardiovascular en estudiantes de la Universidad Santo Tomás. Revista chilena de nutrición, 42(1), 70-76.

12. Bonita, R., De Courten, M., Dwyer, T., Jamrozik, K., \& Winkelmann, R. (2001). Vigilancia de los factores de riesgo para enfermedades no transmisibles: el método progresivo de la OMS. Ginebra: Organización Mundial de la Salud.

13.Ministerio de salud.(1993). Resolución número 8414 de 1993. Recuperado en: https://www.minsalud.gov.co/sites/rid/Lists/BibliotecaDigital/RIDE/DE/ DIJ/RESOLUCION -8430-DE-1993.

14. Manzini, J. L. (2000). Declaración de Helsinki: principios éticos para la investigación médica sobre sujetos humanos. Acta bioethica, 6(2), 321-334. Recuperado en: https://scielo.conicyt.cl/ $\mathrm{s} \mathrm{c}$ i e l o $\mathrm{p} h \mathrm{p}$ ? pid $=S 1726-569 \times 2000000200010 \&$ script $=$ sci_arttext

15. Organización Mundial de la Salud. (en prensa) Enfermedades cardiovasculares, nota informativa; enero 2011. Recuperado en: https://www.paho.org/ $\mathrm{h} \mathrm{q} / \mathrm{i} \mathrm{n} \quad \mathrm{d}$ e $\mathrm{x}$. $\mathrm{p}$ h $\mathrm{p}$ ? option=com_content\&view=article\&id=10366:2015ncds-16-million-lives-who-urges-more-action\&ltemid $=135$ \&lang $=e s$

16. Mollinedo Montaño, F. E., Trejo Ortiz, P. M., Araujo Espino, R., \& Lugo Balderas, L. G. (2013). Índice de masa corporal, frecuencia y motivos de estudiantes universitarios para realizar actividad física. Educación Médica Superior, 27(3), 189-199. Recuperado en: http://www.ems.sld.cu/index.php/ ems/article/view/218/107

17. Salazar Vázquez, B., Salazar Vázquez, M., y Pérez Tamayo, R. (2015) ¿Gorditos o enfermos? La obesidad en niños y adolescentes [Internet]. Distrito Federal: FCE - Fondo de Cultura Económica; [cited 2018 May 9].Recuperado en: ProQuest Ebook Central.

18. Moreno-Bayona, J. A. (2018). Niveles de sedentarismo en estudiantes universitarios de pre- 
grado en Colombia. Revista Cubana de Salud Pública, 44, e881. Recuperado en: http://www.revsaludpublica.sld.cu/index.php/spu/article/view/ $881 / 1135$.

19. Acuña-Medina, L., Arias-Quispe, Y., ChipanaCollahua, D., Sifuentes-Gómez, L., \& Quispe-Rosales, P. (2018). Exceso de peso y actividad física en estudiantes de enfermería de una universidad del Norte de Lima. Revista Peruana de Salud Pública y Comunitaria, 1(1), 26-29. Recuperado en: http://revistas.ual.edu.pe/index.php/revistaual/article/view/36/17.
20. Fajardo, E., Camargo, Y., Buitrago, E., Peña, L., \& Rodríguez, L. (2016). Estado nutricional y preferencias alimentarias de una población de estudiantes universitarios en Bogotá. Revista Med, 24(2), 58-65. Recuperado en: http://dx.doi.org/10.18359/rmed.2641.

21. Organización Mundial de la salud. (en prensa) Enfermedades Cardiovasculares control y prevención. Recuperado en: https://www.who.int/cardiovascular_diseases/es/ 


\section{TABLAS}

Tabla 1. Características sociodemográficas de los estudiantes de las ciencias de la salud.

\begin{tabular}{|c|c|c|c|c|c|c|}
\hline Sexo & Medicina & Enfermería & Fisioterapia & $\begin{array}{l}\text { Instrumentación } \\
\text { Quirúrgica }\end{array}$ & Total & $\begin{array}{l}\text { Chi- } \\
\text { cuadrado }\end{array}$ \\
\hline Femenino & 48 [55\%] & 63 [79\%] & 50 [68\%] & 39 [74\%] & 200 [68\%] & \multirow{2}{*}{11,7} \\
\hline Masculino & 39 [45\%] & 17 [21\%] & 24 [32\%] & 14 [26\%] & 94 [32\%] & \\
\hline Edad & Medicina & Enfermería & Fisioterapia & $\begin{array}{l}\text { Instrumentación } \\
\text { Quirúrgica }\end{array}$ & Total & $\begin{array}{l}\text { Chi- } \\
\text { cuadrado }\end{array}$ \\
\hline $15-19$ & 31 [36\%] & $39[49 \%]$ & 29 [39\%] & 18 [34\%] & 116 [39\%] & \multirow{4}{*}{8,00} \\
\hline $20-24$ & 48 [55\%] & 35 [44\%] & 40 [54\%] & 29 [55\%] & 152 [52\%] & \\
\hline $25-29$ & $3[3 \%]$ & 4 [5\%] & $4[5 \%]$ & $3[6 \%]$ & 14 [5\%] & \\
\hline 30-mas & $5[6 \%]$ & 2 [3\%] & $1[1 \%]$ & $3[6 \%]$ & $11[4 \%]$ & \\
\hline Procedencia & Medicina & Enfermería & Fisioterapia & $\begin{array}{l}\text { Instrumentación } \\
\text { Quirúrgica }\end{array}$ & Total & $\begin{array}{l}\text { Chi- } \\
\text { cuadrado }\end{array}$ \\
\hline Urbana & 55 [63\%] & $62[78 \%]$ & 70 [95\%] & $33[62 \%]$ & 220 [75\%] & \multirow{2}{*}{23,6} \\
\hline Rural & 32 [37\%] & 18 [23\%] & 4 [5\%] & 20 [38\%] & 74 [25\%] & \\
\hline Estado civil & Medicina & Enfermería & Fisioterapia & $\begin{array}{l}\text { Instrumentación } \\
\text { Quirúrgica }\end{array}$ & Total & $\begin{array}{l}\text { Chi- } \\
\text { cuadrado }\end{array}$ \\
\hline Casado & $4[5 \%]$ & $4[5 \%]$ & $4[5 \%]$ & $3[6 \%]$ & 15 [5\%] & \multirow[t]{5}{*}{10,9} \\
\hline Divorciado & $0[0 \%]$ & 0 [0\%] & $0[0 \%]$ & $1[2 \%]$ & $1[0 \%]$ & \\
\hline Viudo & $1[1 \%]$ & $0[0 \%]$ & $0[0 \%]$ & $0[0 \%]$ & $1[0 \%]$ & \\
\hline Unión libre & 8 [9\%] & $8[10 \%]$ & $6[8 \%]$ & $9[17 \%]$ & $31[11 \%]$ & \\
\hline Soltero & 74 [85\%] & $68[85 \%]$ & 64 [86\%] & 40 [75\%] & 246 [84\%] & \\
\hline
\end{tabular}

Fuente: Consolidado de aplicación de instrumento del proyecto 
ESTILOS DE VIDA RELACIONADOS CON FACTORES DE RIESGO CARDIOVASCULAR EN ESTUDIANTES CIENCIAS DE LA SALUD

Tabla 2. Factores de riesgos relacionados con los estilos de vida de los estudiantes de las ciencias de la salud.

\begin{tabular}{|c|c|c|c|c|c|c|}
\hline $\begin{array}{l}\text { Frecuencia de consumo } \\
\text { de tabaco }\end{array}$ & Medicina & Enfermería & Fisioterapia & $\begin{array}{l}\text { Instrumentación } \\
\text { Quirúrgica }\end{array}$ & Total & Chi-cuadrado \\
\hline Siempre & $1[1,1 \%]$ & $0[0,0 \%]$ & $2[4,1 \%]$ & $0[0,0 \%]$ & $3[1,0 \%]$ & \multirow[t]{4}{*}{8,37} \\
\hline Frecuentemente & $1[1,1 \%]$ & $1[1,3 \%]$ & $0[0,0 \%]$ & $2[3,8 \%]$ & $4[1,3 \%]$ & \\
\hline Algunas Veces & $4[4,6 \%]$ & $5[6,3 \%]$ & $7[8,1 \%]$ & $4[7,5 \%]$ & $20[6,8 \%]$ & \\
\hline Nunca & $81[93,1 \%]$ & $74[28,0 \%]$ & $65[87,8 \%]$ & $47[88,7 \%]$ & $267[90,8 \%]$ & \\
\hline $\begin{array}{l}\text { Frecuencia de consumo } \\
\text { de alcohol }\end{array}$ & Medicina & Enfermería & Fisioterapia & $\begin{array}{l}\text { Instrumentación } \\
\text { Quirúrgica }\end{array}$ & Total & Chi-cuadrado \\
\hline Siempre & $0[0,0 \%]$ & $0[0,0 \%]$ & $3[4,1 \%]$ & $0[0,0 \%]$ & $3[1,0 \%]$ & \multirow[t]{4}{*}{22,8} \\
\hline Frecuentemente & $5[5,7 \%]$ & $2[2,5 \%]$ & $2[2,7 \%]$ & $5[9,4 \%]$ & $14[4,7 \%]$ & \\
\hline Algunas Veces & $20[23,3 \%]$ & $12[15,0 \%]$ & $25[35,1 \%]$ & $9[17,0 \%]$ & $66[22,4 \%]$ & \\
\hline Nunca & $62[71,3 \%]$ & $66[82,5 \%]$ & $44[58,1 \%]$ & $39[58,1 \%]$ & $211[71,7 \%]$ & \\
\hline $\begin{array}{l}\text { Frecuencia de las } \\
\text { prácticas de ejercicio }\end{array}$ & Medicina & Enfermería & Fisioterapia & $\begin{array}{l}\text { Instrumentación } \\
\text { Quirúrgica }\end{array}$ & Total & Chi-cuadrado \\
\hline Siempre & $5[5,7 \%]$ & $9[11,3 \%]$ & $6[8,1 \%]$ & $2[3,8 \%]$ & $22[7,4 \%]$ & \multirow[t]{4}{*}{13,3} \\
\hline Frecuentemente & $17[19,5 \%]$ & $5[6,3 \%]$ & $7[8,1 \%]$ & $10[18,9 \%]$ & $39[13,2 \%]$ & \\
\hline Algunas Veces & $24[27,6 \%]$ & $21[26,3 \%]$ & $21[29,7 \%]$ & $19[35,8 \%]$ & $85[28,9 \%]$ & \\
\hline Nunca & $41[47,1 \%]$ & $45[56,3 \%]$ & $40[54,1 \%]$ & $22[41,5 \%]$ & $148[50,3 \%]$ & \\
\hline
\end{tabular}

Fuente: Consolidado de aplicación de instrumento del proyecto 
ESTILOS DE VIDA RELACIONADOS CON FACTORES DE RIESGO CARDIOVASCULAR EN ESTUDIANTES CIENCIAS DE LA SALUD

Tabla 3. Hábitos alimentarios de los estudiantes de las ciencias de la salud.

\begin{tabular}{|c|c|c|c|c|c|c|}
\hline $\begin{array}{l}\text { Frecuencia del } \\
\text { consumo de comidas } \\
\text { ricas en grasas }\end{array}$ & Medicina & Enfermería & Fisioterapia & $\begin{array}{l}\text { Instrumentación } \\
\text { Quirúrgica }\end{array}$ & Total & $\begin{array}{l}\text { Chi- } \\
\text { cuadrado }\end{array}$ \\
\hline Siempre & $9[10,3 \%]$ & $8[10,0 \%]$ & $8[10,8 \%]$ & $7[13,2 \%]$ & $32[10,8 \%]$ & \multirow[t]{4}{*}{5,05} \\
\hline Frecuentemente & $26[29,9 \%]$ & $28[35 \%]$ & $24[32,4 \%]$ & $21[39,6 \%]$ & $99[33,6 \%]$ & \\
\hline Algunas Veces & $46[52,9 \%]$ & $37[46,3 \%]$ & $39[52,7 \%]$ & $20[37,7 \%]$ & $142[48,2 \%]$ & \\
\hline Nunca & $6[6,9 \%]$ & $7[8,8 \%]$ & $3[4,1 \%]$ & $5[9,4 \%]$ & $21[7,1 \%]$ & \\
\hline $\begin{array}{l}\text { Frecuencia del } \\
\text { consumo de gaseosa } \\
\text { a la semana }\end{array}$ & Medicina & Enfermería & Fisioterapia & $\begin{array}{l}\text { Instrumentación } \\
\text { Quirúrgica }\end{array}$ & Total & $\begin{array}{l}\text { Chi- } \\
\text { cuadrado }\end{array}$ \\
\hline Siempre & $19[21,8 \%]$ & 13 [ 16,3\%] & $12[16,2 \%]$ & $16[30,2 \%]$ & $60[20,4 \%]$ & \multirow[t]{4}{*}{14,64} \\
\hline Frecuentemente & 26 [29,9\%] & 22 [27,5\%] & 19 [25,7\%] & $18[34,0 \%$ & $85[28,9 \%]$ & \\
\hline Algunas Veces & $23[26,4 \%]$ & 33 [41,3\%] & 33 [44,6\%] & $15[28,3 \%]$ & $104[35,4 \%]$ & \\
\hline Nunca & 19 [21,8\%] & $12[15,0 \%]$ & $10[13,5 \%]$ & $4[7,5 \%]$ & $45[15,3 \%]$ & \\
\hline $\begin{array}{l}\text { Frecuencia del } \\
\text { consumo de bebidas } \\
\text { energizantes }\end{array}$ & Medicina & Enfermería & Fisioterapia & $\begin{array}{l}\text { Instrumentación } \\
\text { Quirúrgica }\end{array}$ & Total & $\begin{array}{l}\text { Chi- } \\
\text { cuadrado }\end{array}$ \\
\hline Siempre & $13[14,9 \%]$ & $11[13,8 \%]$ & 17 [23,0\%] & $7[13,2 \%]$ & $48[16,3 \%]$ & \multirow[t]{4}{*}{6,50} \\
\hline Frecuentemente & $24[27,6 \%]$ & $24[30,0 \%]$ & 15 [20,3\%] & $15[28,3 \%]$ & 78 [26,5\%] & \\
\hline Algunas Veces & $28[32,2 \%]$ & $23[28,7 \%]$ & 27 [36,5\%] & $20[37,7 \%]$ & $98\{33,3 \%]$ & \\
\hline Nunca & $22[25,3 \%]$ & 22 [ $27,5 \%]$ & 15 [20,3\%] & $11[20,8 \%]$ & $70[23,8 \%]$ & \\
\hline $\begin{array}{l}\text { Frecuencia del } \\
\text { consumo de comidas } \\
\text { rápidas a la semana }\end{array}$ & Medicina & Enfermería & Fisioterapia & $\begin{array}{l}\text { Instrumentación } \\
\text { Quirúrgica }\end{array}$ & Total & $\begin{array}{l}\text { Chi- } \\
\text { cuadrado }\end{array}$ \\
\hline Siempre & $11[1.6 \%]$ & $4[5 \%]$ & $3[4,1 \%]$ & $10[18,9 \%]$ & $28[9,5 \%]$ & \multirow[t]{4}{*}{20,8} \\
\hline Frecuentemente & $16[18,4 \%]$ & $14[17,5 \%]$ & $14[18,9 \%]$ & 17 [32.1\%] & $61[[20,7 \%]$ & \\
\hline Algunas Veces & $43[49,4 \%]$ & $42[52,5 \%]$ & $43[58,1 \%]$ & $22[41,5 \%]$ & $150[51.0 \%]$ & \\
\hline Nunca & $17[19,5 \%]$ & 20 [25,0\%] & 14 [18,9\%] & $4[7,5 \%]$ & $55[18,7 \%]$ & \\
\hline
\end{tabular}

Fuente: Consolidado de aplicación de instrumento del proyecto 